\title{
GENERAL
}

\section{'IF I KNEW THAT IT COULD HELP...' - FACTORS INFLUENCING ADHERENCE TO TREATMENT AFTER MYOCARDIAL INFARCTION - RESULTS FROM A QUALITATIVE STUDY GUIDED BY HEALTH BELIEF MODEL}

\author{
AGNIESZKA LISOWSKA ${ }^{1}$, ANNA M. DWORAKOWSKA ${ }^{1 *}$, AGNIESZKA SERAFIN ${ }^{2}$ \\ and MAŁGORZATA KOZŁOWSKA-WOJCIECHOWSKA ${ }^{1}$
}

${ }^{1}$ Department of Clinical Pharmacy and Pharmaceutical Care, Medical University of Warsaw, ul. Banacha 1, 02-097 Warsaw, Poland. ${ }^{2} 1$ st Chair and Department of Cardiology, Medical University of Warsaw, ul. Banacha 1a, 02-097 Warsaw, Poland.

\begin{abstract}
Mortality after myocardial infarction among patients at the age of professional activity is at least 2 -fold higher in Poland than in other European Union countries. Six months after myocardial infarction, high adherence is maintained on average by every fourth patient. Therefore, the aim of the study was to explore factors possibly affecting adherence to pharmacological and non-pharmacological treatment among young myocardial infarction patients with the use of the Health Belief Model. Twenty face-to-face or telephone interviews were conducted, recorded, and transcribed verbatim. Directed content analysis was used to analyze the collected data. Participants (15 male and 5 female, mean age: 49.7, mean time from myocardial infarction: 2.65 years) have reported many obstacles to adherence to recommended treatment, especially non-pharmacological. Many interviewees denied their increased susceptibility to subsequent cardiovascular events, neglecting the severity of the impact of myocardial infarction on future health. Moreover, gains from non-pharmacological treatment were rarely acknowledged. Neglecting and denying the disease, together with little perceived benefits resulting from non-pharmacological treatment, and with a large number of obstacles, may lead to inadequate patient adherence to treatment. The obtained results are consistent with the assumption of the HBM.
\end{abstract}

Keywords: myocardial infarction, treatment adherence, non-pharmacological, Poland, qualitative research

Ischemic heart disease, including myocardial infarction (MI), is the main cause of death among subjects below 65 years of age in Europe. Although young people are the minority of patients with MI, the mortality rate after acute MI among patients at the age of 25-64 years is over 2.5-fold higher for men and over 2-fold higher for women in Poland than in other European Union countries (1).

In Poland patients after MI often fail to adhere to therapeutic recommendations, with only every fourth maintaining a high adherence to prescribed medicines 6 months after the MI, and almost the same percentage of patients showing a low adherence (2). Throughout one year after the MI, only $29 \%$ of patients adhered sufficiently to three pivotal groups of medicines (3).

Non-adherence is a global problem. There is a steady rate of discontinuation of taking medicines throughout the follow-up period after MI, with more than $50 \%$ of patients stopping taking medicines altogether 3 years after MI $(4,5)$. The situation regarding non-pharmacological adherence is even worse $(6,7)$. According to studies conducted in France, only $10 \%$ of patients adhere to recommendations regarding pharmacotherapy and modification of the lifestyle by undertaking certain physical activity and quitting smoking cigarettes 6 years after the MI (8).

Patient adherence may be discussed with regard to numerous theoretical models. Health Belief Model (HBM) is one of the most frequently used models to describe and predict health behavior. In light of the HBM, taking up health activities depends on how the patient perceives his/her susceptibility to a given disease or its consequences and severity of the condition, which together determine

* Corresponding author: e-mail: anna.dworakowska@wum.edu.pl 
the perceived threat to health. At the same time, the patient evaluates the attractiveness of a given health behavior by assessing the expected benefits and barriers. Undertaking and maintaining health behavior is supported by the patient's conviction about his/her self-efficacy (judgment of capabilities to execute a given activity required to achieve given results) (9). Previous studies showed a successful application of the HBM in explaining and predicting preventive health behavior, including treatment adherence $(10,11,12,13,14)$. However, there is a paucity of qualitative data, which deeper explore factors influencing adherence among MI patients. Therefore, the study aims to identify the factors possibly influencing the adherence of young (55 years or younger at the time of MI) people after MI to both pharmacological and non-pharmacological treatment, based on the HBM constructs.

\section{METHODS}

The study was conducted after receiving approval from the Ethics Committee at the Medical University of Warsaw, Poland, No. AKBE/143/17. The face-to-face or telephone interviews, depending on the patient's preference, were held by AL among a convenience sample of 20 individuals. The openended questions in the semi-structured interview were based on the HBM, the life experience of patients after MI, and literature reviews, and consulted with a qualified psychologist, who works with cardiac patients.

Participants were enrolled out of young subjects ( $\leq 55$ years at the age of MI) hospitalized at a tertiary university hospital in Warsaw (Poland), on the basis of medical records. Hospitalization due to MI took place in a period of up to five years before the study.

Each participant was informed about the assumptions and aims of the study and that the conversation would be recorded. Each gave their informed consent to participate in the study.

The interviews were recorded and transcribed verbatim immediately after conducting the interview. Then directed content analysis following the HBM constructs was conducted (15). NVivo 12.2 Pro QRS International software was used to facilitate the analysis. The transcripts were coded using five HBM constructs. Two researchers [AL and AD] were independently coding the transcripts and any disagreements between researchers were discussed until an agreement was reached. Among each construct, some categories (factors) have been coded, which are presented in Tables 1 and 2. The inter-

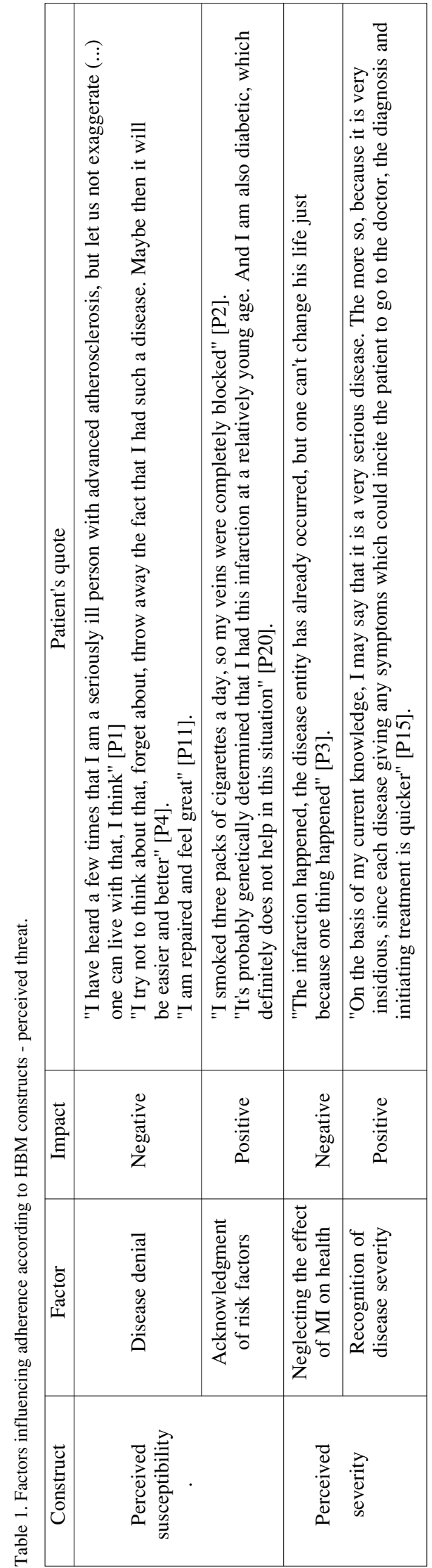




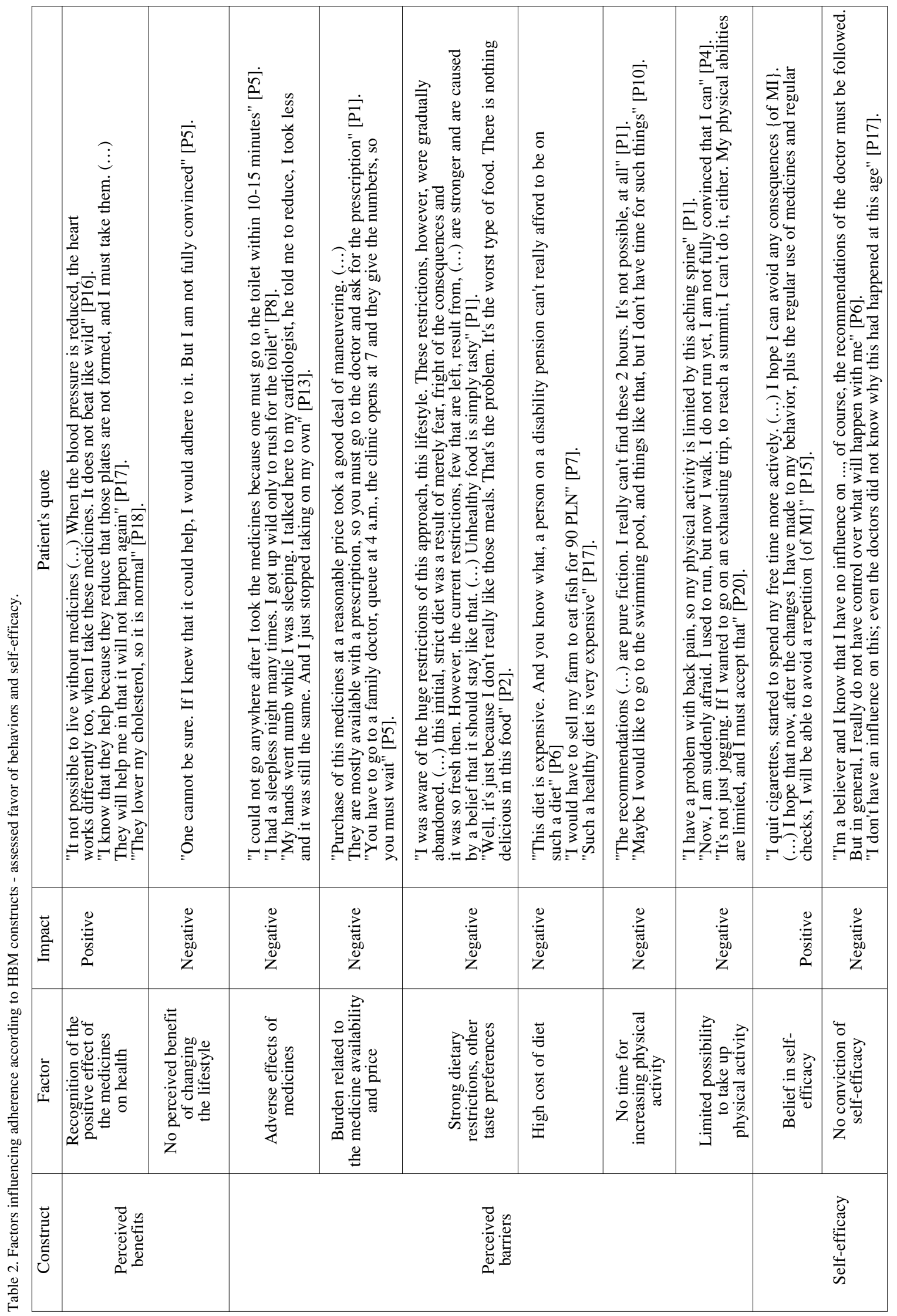


views started on 27/04/2018 and were continuing until data saturation was reached, meaning that in the next interview no new factor emerged. The last interview was conducted on 22/10/2018.

\section{RESULTS}

The group of 20 participants (median age: 50 years; range: $42-58$ /at the day of interview/) included $75 \%(n=15)$ of men. The time between MI and the interview was on average 2.65 years (range: 1.5 $5)$. Most of the participants were married $(n=16$; $80 \%)$ and employed $(n=13 ; 65 \%)$. The participants were coded and their interviews were identified based on the assignment of the letter ' $\mathrm{P}$ ' and a series of numbers [P1, P2, etc.]. A conceptual model of factors influencing adherence based on HBM is presented in Figure 1. Factors influencing adherence according to HBM constructs, with patients' quotes, are presented in Table 1 and Table 2, includes the following.

\section{Perceived susceptibility}

Participants presented two different attitudes regarding perceived susceptibility to subsequent cardiovascular (CV) events. Some did not want to think about their increased risk of cardiovascular diseases (CVD). Despite their past MI and potential risk factors of CVD, they denied the disease and tried to neglect possible negative consequences of MI, treating it as an incident without further effect on health. However, a small group of participants already before the MI recognized its risk factors, e.g. diabetes, so they perceived themselves as susceptible to CVD and were aware of the possibility of recurrent CV events.

\section{Perceived severity}

Interviewees rarely perceived MI and its consequences as a serious disease. Only two individuals talked about MI and underlying atherosclerosis as a serious disease. In the context of MI consequences, participants were most afraid of disability, understood as the inability to work.

\section{Perceived benefits}

The majority of participants acknowledged taking medicines as essential in secondary prevention. Most expected the medicines to make them live longer and some were convinced that it will prevent recurrent $\mathrm{CV}$ events. However, participants were very rarely aware of the gains of non-pharmacological treatment. Some were not at all convinced about its benefits.

\section{Perceived barriers}

Most reported obstacles to adherence were related to the modification of lifestyle, especially dietary restriction. Importantly, at least during the first months after the MI, the majority of participants tried to eat a healthy diet. They were mostly driven by fear of another CV event. However, the restrictions concerning e.g. reduced consumption of fat, sugar, and meat, and increased consumption of vegetables, were followed only for a few months by some participants, and then (after 3-12 months) they returned to their previous dietary habits. They perceived such modification as very restricting their life. Therefore, as time passed after the MI, they reduced the number of recommendations they had followed. Smoking cessation was a difficult change to implement. Significantly, out of six patients, who admitted to having been smoking before MI, two

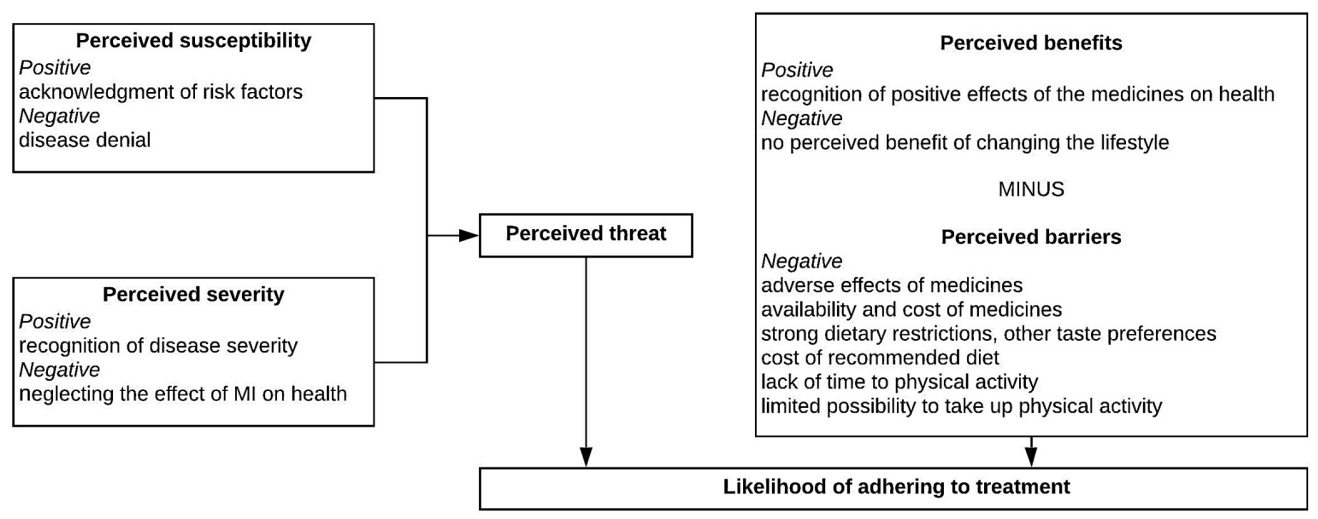

Figure 1. Conceptual model of factors influencing adherence based on HBM. 
returned to the habit, explaining it as arising from stress-related to returning to work.

Concerning physical activity, the main obstacles were lack of time among individuals returning to professional activity and a limited possibility to take up physical exercises, resulting from both physical consequences of MI and mental barrier - fear after the MI.

Frequently reported barriers related to medicines were: cost, which was a burden especially for participants being on sick leave or disability pension, having reduced income; necessity to visit a doctor to obtain a prescription, connected with difficulties related to the healthcare system and a long waiting time for the visit; and adverse effects of medicines, resulting in reduced life activity of participants.

\section{Self-efficacy}

Few interviewees felt that undertaken activities were able to reduce the risk factors of CVD. Some were convinced that they were not able to modify their lifestyle according to the recommendations because of 'weak will'. Others felt that they had no influence on their health state at all. Sometimes this was a result of religious beliefs.

Patients attached the highest importance to taking medicines. However, this was mostly a result of external motivation (the doctor's instruction on taking medicines), not of inner conviction of self-efficacy.

\section{DISCUSSION AND CONCLUSION}

In light of the HBM, the reasons for inadequate adherence may be found in perceiving MI as having little impact on further health (perceived low severity) and neglecting one's predisposition to recurrent $\mathrm{CV}$ events (perceived low susceptibility). In the present study, most participants represented both attitudes. Moreover, many barriers to the therapeutic recommendations recognized by participants and perceived low benefits could also reduce the likelihood of introducing and maintaining recommended activities.

Participants adhered or did not adhere to various elements of therapy, including taking medications, diet modification, and physical activity, depending on the importance attached to them. This observation is consistent with the assumptions of the HBM that a patient's adherence depends on a perceived health threat and assessed favors. Denying and neglecting the disease, which was observed among studied participants, is a rarely mentioned factor affecting adherence. These elements, which exemplify low perceived susceptibility, were proven to be associated with nonadherence (16). Denying the disease and its influence on health is a method of handling the past MI. However, its effect on future health behaviors changes with time. Initially, it helps to handle fear caused by the disease, but in the long perspective, it may delay treatment, lead to poor knowledge of one's disease, and non-adherence (17).

However, perceived threat (susceptibility and severity of the disease and its consequences) refers to the health condition, only perceived benefits and barriers may differ among pharmacological and non-pharmacological treatment. Hence, the differences in adherence to each therapeutic recommendation can result from a variant perception of the latter two constructs. Moreover, perceived benefits and barriers are the strongest predictors of health behaviors (10). Therefore, the differences among adherence to pharmacological and non-pharmacological treatment may be significant.

Most of the participants were not aware of the benefits of diet modification and adequate physical activity, therefore non-pharmacological adherence declared by participants was very low. The knowledge of benefits positively impacts adherence (18, 19). Thus, patients should be educated about the proven benefits resulting from lifestyle modification after MI, such as reducing the risk of mortality (20).

European Society of Cardiology (ESC) highlights the critical importance of collaborative care of MI patients recommending (Class I Level A) the involvement of multidisciplinary healthcare professionals (cardiologists, general practitioners, nurses, dieticians, physiotherapists, psychologists, pharmacists) in MI patient management (21). Pharmacists' interventions (e.g. patient education and counseling) improve medication adherence, lifestyle modifications, and control of modifiable risk factors in postMI patients $(22,23)$.

Obstacles related to the medicines, mostly resulting from adverse effects, were usually not very burdensome for participants. This may be the explanation of moderate and high adherence to taking medicines declared by most participants. However, using only medicines at an adequately high level does not lead to achieving the set treatment aims in numerous patients (24). Therefore, long-term care of cardiac patients should emphasize the necessity of non-pharmacological adherence, highlighting the benefits of such behavior.

In the studied population, both positive and negative factors influencing adherence were identified among most HBM constructs. As the recent 
study shows, the impact of perceived susceptibility and severity on adherence may be opposite than predicted by the HBM (25). Thus, the direction of their impact on health behaviors among patients with MI should be assessed in further researches.

Due to the qualitative type of the study, the power of influence of the HBM constructs on adherence to treatment recommendations has not been studied. Additional researches should be conducted to assess this power.

This study has some limitations. The sample size was relatively small and participants were recruited from one hospital in the capital city. Therefore, caution about generalizing findings to other populations is needed. Although the small sample size is appropriate for a qualitative approach, it is possible that not all probable factors influencing adherence were revealed. Most participants claimed they take their medicines. These are self-reported data based only on a simple declaration, which means they may be overestimated.

The conducted study shows that taking medicines is not the most burdensome problem for the patients, but lifestyle modifications are much more difficult to introduce. Non-adherence, especially regarding non-pharmacological treatment, of young patients after MI may result not only from neglecting and denying the disease, but also from too little benefits attached to these activities by the patients, and a large number of obstacles while implementing lifestyle modifications. This finding is consistent with the assumption of the HBM.

\section{Funding source}

This study was funded by the Medical University of Warsaw (Warsaw, Poland), grant number FW7/PM1/18.

\section{Acknowledgments}

We thank all the patients with MI who participated in the interviews, for their contribution to the study.

\section{Conflicts of interest}

The authors declare no conflict of interest.

\section{REFERENCES}

1. WHO Report, Społeczne nierówności w zdrowiu $\mathrm{w}$ Polace [Social inequalities in health in Poland], WHO 2012 (in Polish).
2. Kosobucka A., Michalski P., Pietrzykowski Ł., Kasprzak M., Obońska K., et al.: Patient Prefer. Adherence 12, 333 (2018).

3. Pietrzykowski Ł., Michalski P., Kosobucka A., Kasprzak M., Fabiszak T., et al.: Sci. Rep. 10, 12028 (2020).

4. Mathews R., Wang T.Y., Honeycutt E., Henry T.D., Zettler M., et al.: Am. Heart J. 170, 62 (2015).

5. Shah N.D., Dunlay S.M., Ting H.H., Montori V.M., Thomas R.J., et al.: Am. J. Med. 122, 961.e7-13 (2009).

6. Sudevan R., Vasudevan D., Raj M., Thachathodiyl R., Vijayakumar M., et al.: . BMJ Open. 10, 10.e037618 (2020).

7. Kotseva K., De Backer G., De Bacquer D., Rydén L., Hoes A., et al.: Eur. J. Prev. Cardiol. 26, 824 (2019).

8. Dibao-Dina C., Angoulvant D., Lebeau J.P., Peurois J.E., Abdallah El Hirtsi A., et al.: PLoS One. 13, e0202986 (2018).

9. Champion V.L., Skinner C.S.: The Health Belief Model, in Health behavior and health education. Theory, Research and Practice. Glanz K., Rimer B., Viswanath K. Ed., 4th ed. pp. 45-65, Jossey-Bass, San Francisco 2008.

10. Carpenter C.J.: Health Commun. 25, 661 (2010).

11. Kirag N., Kizılkaya M.: BMC Womens Health. 19, 132 (2019).

12. Wang X., Chen D., Xie T., Zhang W.: J Obstet Gynaecol Res. 45, 2440 (2019).

13. Tong K.K., Chen J.H., Yu E.W., Wu A.M.S.: Appl. Psychol. Health Well Being.

14. Lilly F.R.W., Jun H.J., Alvarez P., Owens J., Malloy L., et al.: Brain Behav. e01873 (2020).

15. Hsieh H.F., Shannon S.E.: Qual Health Res. 15, 1277 (2005).

16. Ganasegeran K., Rashid A.: Patient Prefer. Adherence 11, 1975 (2017).

17. Fowers B.J.: J Psychosom Res. 36, 469 (1992).

18. Kamran A., Sadeghieh Ahari S., Biria M., Malepour A., Heydari H.: Ann Med Health Sci Res. 4, 922 (2014).

19. Khoshbakht Pishkhani M., Dalvandi A., Ebadi A., Hosseini M.: J Vasc Nurs. 37, 264 (2019).

20. Booth J.N. 3rd, Levitan E.B., Brown T.M., Farkouh M.E., Safford M.M., et al.: Am. J Cardiol. 113, 1933 (2014).

21. Collet J.P., Thiele H., Barbato E., Barthélémy O., Bauersachs J., et al.: Eur Heart J. ehaa575 (2020).

22. Sundararajan S., Thukani Sathanantham S., Palani S.: Curr Ther Res Clin Exp. 92, 100577 (2020). 
23. Chiou C.C., Tsai T.H., Lee C.H., Lin C.J., Chung W.J., et al.: Acta Cardiol Sin. 35, 290 (2019).

C 2020 by Polish Pharmaceutical Society. This is an access article under the CC BY NC license

\section{(http://creativecommons.org/licenses/by-nc/4.0/).}

24. Kotseva K., Wood D., De Bacquer D., De Backer G., Rydén L., et al.: Eur J Prev Cardiol. 23, 636 (2016).

25. Obirikorang Y., Obirikorang C., Acheampong E., Anto E.O., Gyamfi D., et al.: Int J Hypertens. 2018, 4701097 (2018). 